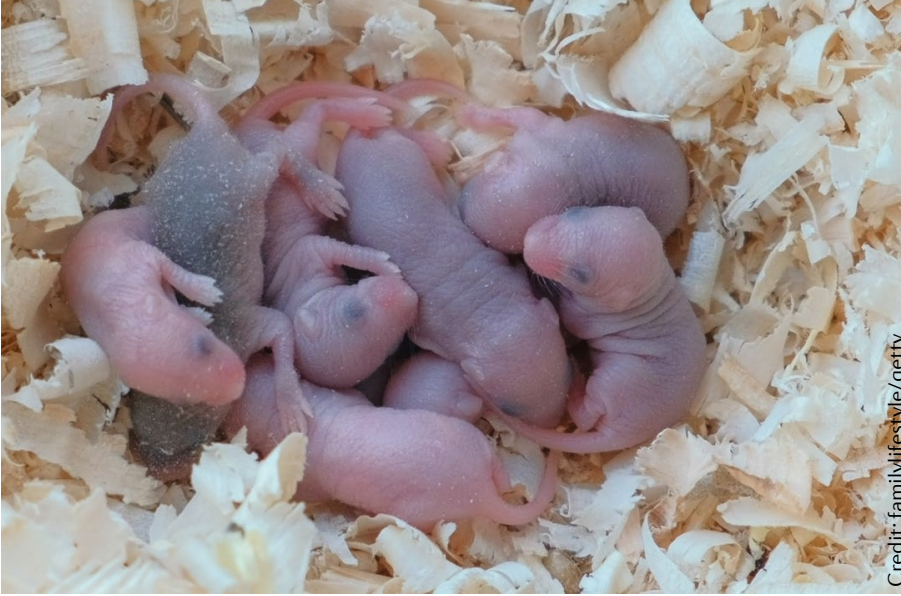

induces ER stress in key tissues involved in energy metabolism and that relieving ER stress perinatally improves metabolic and neurodevelopmental abnormalities in animals born to obese dams."

The team are now planning to investigate non-pharmacological approaches to reducing ER stress in the offspring. "One method we are interested in exploring is by providing the offspring a more balanced diet and/or by making them exercise during early life," concludes Bouret.

Alan Morris

involved

in energy

metabolism

Quantitative X-ray imaging of femurs from wild-type and GIP-GFP-KI mice. BMD has been pseudo-coloured for clarity. Note the lowest BMD in GIP-GFP-KI femur. Image courtesy of Guillaume Mabilleau, Université d'Angers, France.

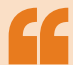

their results support the development of GIP analogues for the treatment of bone disorders featuring ... bone fragility

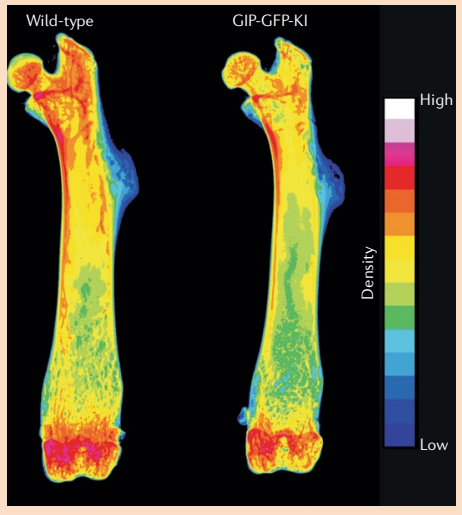

of second-generation GIP analogues, and we are confident that soon we should be able to undertake animal validation of possible candidate molecules," says Mabilleau. The researchers also want to better understand the role of xenin in bone metabolism. "We strongly believe that xenin could be a new target for the development of new innovative molecules for the prevention of bone fragility," concludes Mabilleau.

Claire Greenhill

ORIGINAL ARTICLE Gobron, B. et al. Enteroendocrine K-cells exert complementary effects to control bone quality and mass in mice.J. Bone Miner. Res. https://doi.org/10.1002/jbmr.4004 (2020)

\section{$\beta$-cell dedifferentiation prior to insulitis prevents T1DM}

The mechanisms by which $\beta$-cells contribute to their own destruction in the pathogenesis of type 1 diabetes mellitus (T1DM) are unclear. A study in Cell Metabolism now shows that deletion of the unfolded protein response (UPR) sensor IRE1 $\alpha$ in $\beta$-cells triggers a transient $\beta$-cell dedifferentiation and prevents T1DM in a mouse model.

The UPR enables a cell to adapt to substantial endoplasmic reticulum (ER) stress. However, long-term ER stress can cause the UPR to become pro-apoptotic. Furthermore, the UPR is impaired in preclinical models of T1DM and in humans with T1DM. Using the non-obese diabetic (NOD) mouse model of T1DM, Feyza Engin and colleagues generated a mutant in which IRE1 $a$ was deleted from $\beta$-cells in mouse pups, before the development of insulitis (IRE1 $\alpha^{\beta--}$ NOD mice). Islet morphology and architecture was assessed, islet cells were immunophenotyped and RNA sequencing was carried out on intact islets as well as on single islet cells.

"IRE1 $\alpha$ deletion in NOD $\beta$-cells before insulitis causes a transient dedifferentiation of $\beta$-cells, and these dedifferentiated $\beta$-cells show diminished expression of $\beta$-cell autoantigens and MHC class I molecules, while they increase their expression of immune inhibitory markers," explains corresponding author Engin. Previous work found that deletion of IRE1 $\alpha$ in prenatal or adult $\beta$-cells was associated with a diabetic phenotype. By contrast, the IRE $1 \alpha^{\beta-1-}$ NOD mice showed only transient hyperglycaemia and were protected from T1DM up to 50 weeks of age.

Adoptive transfer experiments were carried out, transferring $T$ cells from IRE $1 \alpha^{\beta---}$ NOD mice or control mice to immunodeficient NOD Rag1 ${ }^{-1-}$ mice. Recipient mice became diabetic 20 weeks after cell transfer if receiving $T$ cells from control mice, whereas they did not develop diabetes mellitus if they received T cells from IRE1 $\alpha^{\beta---}$ NOD mice. "IRE $1 \alpha^{\beta---}$ NOD mice exhibit impaired T cell diabetogenic activity, which leads to protection from autoimmune destruction and diabetes mellitus," says Engin. Of note, the pancreas of IRE $1 \alpha^{\beta-1-}$ NOD mice had substantially reduced numbers of $C D 8^{+} T$ cells compared with control mice. By contrast, no significant alterations were observed in the levels of $\mathrm{CD} 4^{+} \mathrm{T}$ cells, $\mathrm{B}$ cells or macrophages. These findings suggest that modulating the UPR in $\beta$-cells before the immune infiltration can induce $\beta$-cell dedifferentiation that triggers immune tolerance and protects against disease.

"Whether stressed human $\beta$-cells adopt a similar mechanism for stress adaptation and escape from immune attack needs to be tested," concludes Engin. "If this finding is replicated, then there might be a critical therapeutic window for intervention specifically for autoantibody-positive high-risk individuals to briefly alter their $\beta$-cell identity, induce immune tolerance and prevent T1DM."

Shimona Starling

ORIGINAL ARTICLE Lee, H. et al. Beta cell dedifferentiation induced by IRE1a deletion prevents type 1 diabetes. Cell Metab. https://doi.org/10.1016/j.cmet.2020.03.002 (2020)

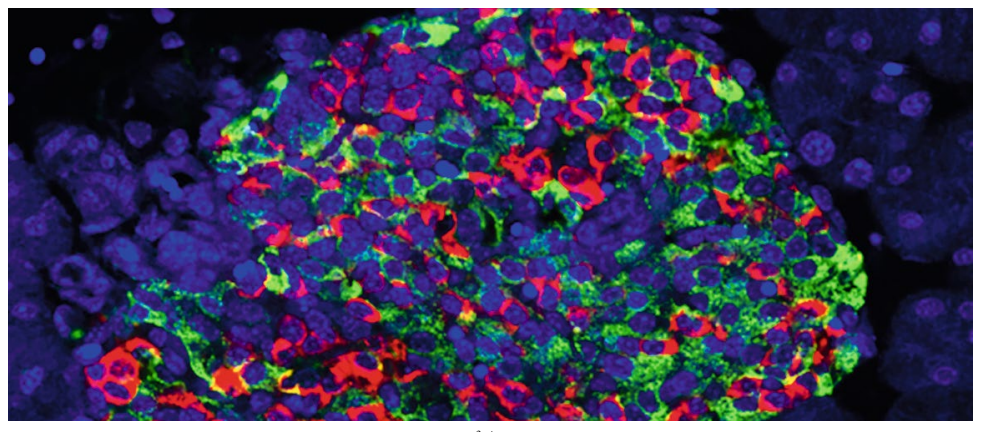

The image shows altered islet composition in IRE1 $\alpha^{\beta-1-}$ non-obese diabetic mice: glucagon-positive cells (red) and insulin-positive cells (green). Image courtesy of Feyza Engin/University of Wisconsin-Madison. 